\title{
The Simulation Research of Radio over PON
}

\author{
Pengfei $\mathrm{Yu}^{1, \mathrm{a}}$ and Xiaojian Zhang ${ }^{1, b}$ \\ ${ }^{1}$ Global Energy Interconnect Research Institute, Nanjing, china \\ ayupengfei@geiri.sgcc.com.cn, ${ }^{\mathrm{b}}$ zhangxiaojian@ geiri.sgcc.com.cn
}

Keywords: Radio-over-PON, power communication access network, Simulation Research.

Abstract. This article starts from the actual demand of Power communication access network construction, and proposes a wireless access method of Radio over PON. The method divides the wireless access system into RRS(Radio Remote System) and RCPC(Radio Central Process Center), which can effectively improve the efficiency of the use of fiber resources, and also can ensure the flexible and reliable transmission of electric power communication data. At last, this article elaborates the simulation system of Radio over PON, which verify the feasibility of the above method.

\section{Introduction}

"We not only need to have a strong carrying capacity and a strong network in the communication network in the core layer, but also need to have a wide range of flexible edge access capabilities in the access layer", which is the China State Grid Corp's clear requirements in the strong smart grid's communication network construction[1].

As the development of smart grid construction, Ethernet passive optical network(EPON) technology is widely used in electric power communication access network. Because the large number of power communication access network terminal nodes distribute widely, the power fiber laying cost is very high. The wireless communication technology has the advantage of low cost and flexibility, so the paper puts forward the technology of Radio-over-PON, which is based on the Ethernet passive optical network technology and wireless communication technology and can balance the communication efficiency, flexibility and security of the Power communication access network.

\section{Typical Power Communication Access Network Architecture}

The current typical Power communication access network architecture is as shown in Fig. 1.

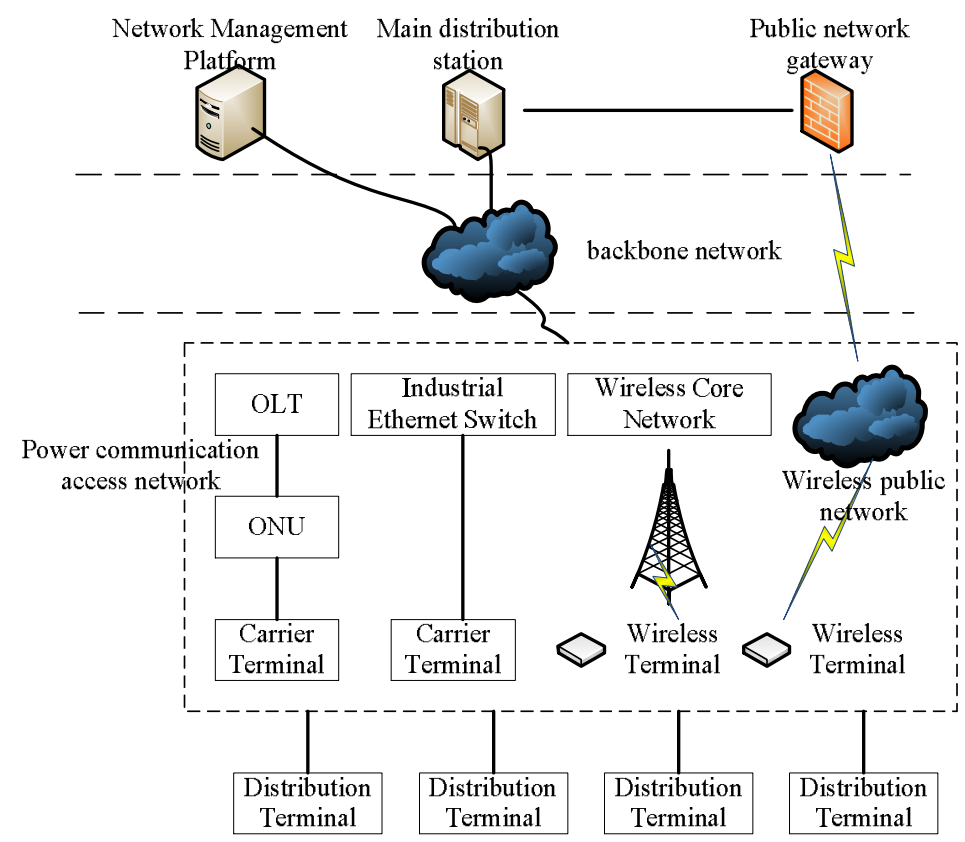

Fig. 1 Typical Power Communication Access Network Architecture 
In the current Power communication network construction, fiber access, wireless access and power line carrier access is main communication access technology. Optical fiber access technology mainly contains industrial Ethernet switch technology and EPON technology, which are carried out in the construction and application. Wireless access technology is mainly carried out by LTE230MHz and LTE1.8GHz as the representative of the local pilot application. Power line carrier communication technology is mainly used in power information collection service.

\section{Radio over PON}

As to reasonably balance the communication efficiency, flexibility and security of the power communication access network, this paper put forward the communication access method on radio over PON[2], as shown in Fig.2. The wireless RF processing unit is integrated with the ONU as the one module. And then the base band signal from the radio frequency processing unit is transmitted to OLT through the EPON system, which is deployed in the substation.

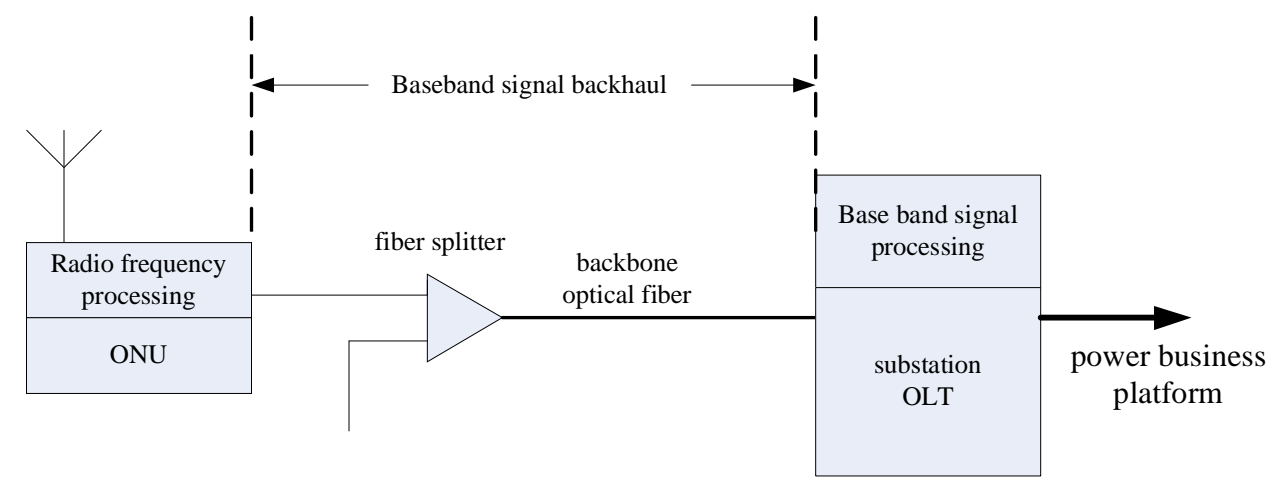

Fig. 2 Communication Access on Radio Over PON

\section{Innovative Interface between RRS and RCPC}

In LTE, for example, the CPRI interface requirements of high bandwidth, low latency. Although at present, the active or passive wave transmission technology can effectively solve this problem, saving the usage amount of fiber, but it will introduce additional transmission facilities, resulting in increased costs.

The sketch map of CPRI(common public radio interface) is as shown in Fig. 3.

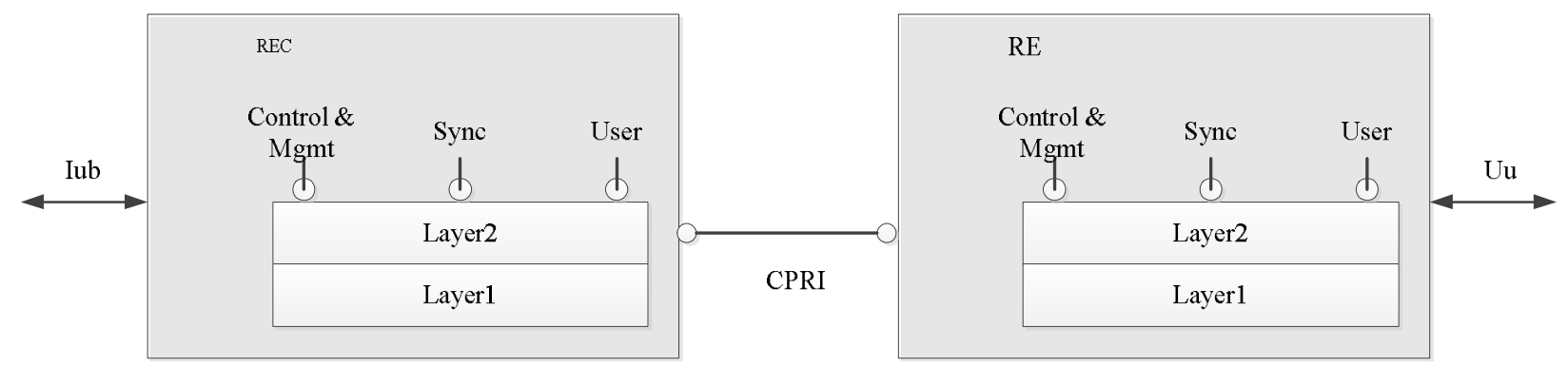

Fig. 3 Common Public Radio Interface[3]

CPRI is a public wireless interface between the REC(radio equipment control center) and RE(radio equipment), which can be transmitted through the cable or twisted pair cable, and can also be transmitted through the optical fiber.

In addition to the user platform data (IQ data), control and management as well as synchronization information must be exchanged between REC and RE. All information flows using the appropriate physical layer (Layer 1) and the data link layer (Layer 2) protocol for multi-channel transmission on a digital continuous communication line. Different information flows through the access to the data link 
layer (Layer 2) by the appropriate service access node. In support of the RE network system structure, CPRI can also be used as a link between the two RE.

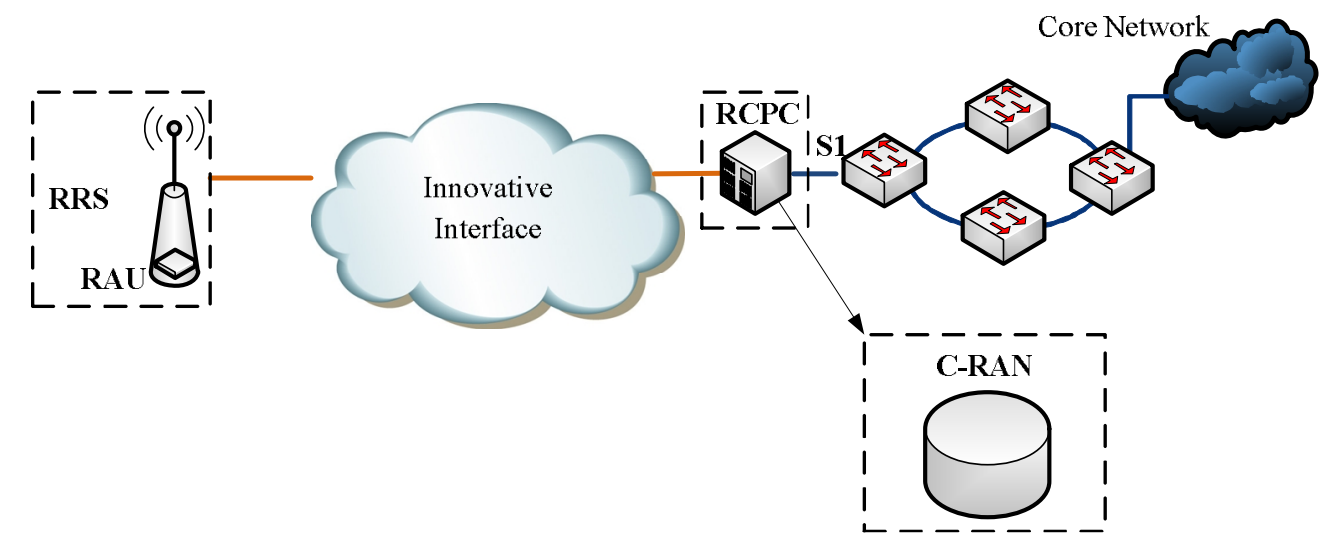

Fig. 4 Innovative Interface between RRS and RCPC

As shown in Fig. 4, the innovative interface divides the wireless access system into RRS and RCPC, which re-defined the front end of the transmission by the point-to-point interface to multipoint-tomultipoint based on Packet switching protocol. Remote radio system includes antenna, a RRU and traditional BBU baseband processing function RAU (radio aggregation unit, RF polymerization unit) and other functions. The RCPC contains the traditional BBU residual function, besides the RAU senior management function etc. The innovative interface implements the function of connecting RRS and RCC, and its interface capability is defined to consider the new requirements of bandwidth, transmission delay, time and frequency synchronization and so on.

\section{Simulation on Radio over PON}

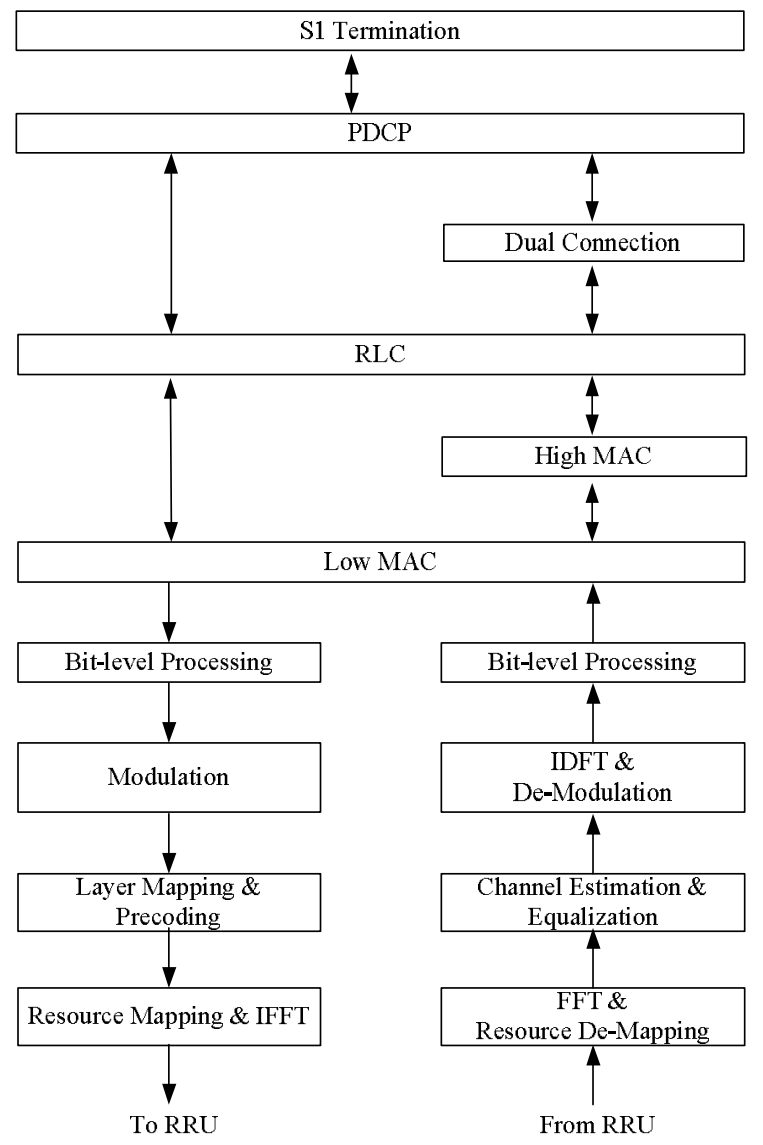

Fig. 5 Simulation Interface between RRS and RCPC 
As shown in Fig. 5, the LTE is an example of the RCC and RRS interface simulation scheme. Hypotheses[4]:

1) 20M LTE carrier;

2) two ports;

3) eight antennas;

4) downlink spectral efficiency for $2 \mathrm{~b} / \mathrm{s} / \mathrm{Hz}$, uplink spectrum efficiency for $1.8 \mathrm{~b} / \mathrm{s} / \mathrm{Hz}$;

5) down at full load the highest modulation level 64QAM, uplink full load when the highest rank modulation 16QAM.

After innovative interface between RRS and RCPC is used, the packet switching delay of the additional switch is introduced, and the time required for the transfer of data is considered. Assuming the most poor transmission conditions, the required one-way data transmission time is t_trans $=500 \mathrm{us}$, then set aside for the BBU processing time of a total of about:

$\mathrm{t} \_$process $=\mathrm{t} \_$total $-2 \times \mathrm{t} \_$trans $=2 \mathrm{~ms}$.

If the transfer time is too low, then the demand for the exchange delay will be too high, resulting in the cost of transmission equipment increased. If the time is left for the baseband processing is too low, it will improve the requirements of the wireless master device.

\section{Conclusions}

At the transmission level, the innovative interface between RRS and RCPC should use packet switch interface in order to facilitate the use of Ethernet transmission, and realize flexible RCPC and RRS among connections, but how to achieve satisfying the delay, delay jitter and synchronization requirements is need further research.

\section{Acknowledgements}

Sponsored by the Innovation Fund Project "The Technology Feasibility Research of Radio over PON based on micro base station", State Grid Smart Grid Research Institute, 2015.

\section{References}

[1] STATE GRID Corporation of China, Development plan of the communication network in 12th Five-Year[R], Beijing, 2010.

[2] Pengfei Yu, Xiaojian Zhang. The Power Communication Access Application of Radio Over PON. The 2015 4th International Conference on Information Technology and Management Innovation (ICITMI 2015).

[3] CPRI, Common Public Radio Interface (CPRI) Specification v6.0, Tech. Rep. Aug. 2013, URL: http://www.cpri.info.

[4] NGMN, Further study on critical C-RAN technologies, April, 2015. 